\title{
Interactive Style of 3D Display of Buildings on Touch Screen
}

\author{
Weina Qu and Xianghong Sun \\ State Key Laboratory of Brain and Cognitive Science, Institute of Psychology, \\ Chinese Academy of Sciences, \\ Beijing 100101, China \\ \{quwn, sunxh\}@psych.ac.cn
}

\begin{abstract}
This study was concentrated on the effect of different building 's floor number display modes and multi-speeds of view changing, for a firefighter recognized the fire scene through the 3D interface display system using a touch screen .The result showed that : (1) Showing number on floors is the worst way. showing numbers on wall, and on two sides of building whatever fixed, or rotating with the building were better than the way of cube display.(2) It's found the condition of "automatic rotation at fixed speed" at 30s was the most helpful for the decision efficiency, "automatic rotation with optional choice" the least helpful. Some mechanism and implications were discussed.
\end{abstract}

Keywords: Fire alarm system; 3D interface display system; Floor-numbering design; 3D display; Rotation.

\section{Introduction}

Fire alarm system is an essential part of high buildings in modern times, which helps firefighters' detection more efficiently and reduces the casualty [1]. The challenge, obviously, is not only make information available to people at any time, at any place, and in any format, but provide "proper" information, at "right" time, in "right" format $[2,3]$. In order to help the firemen to detect the original fire and the current fire spread in a high rise building as quickly and accurately as possible, 3D interface display system, as an important cognitive model [4,5], is necessary for it's convenience [6]. For the convenience of firefighters' decision-making and operation, 3D interface is easier to get the whole spread of fire, especially for vertical spread, very useful for recognizing the features of fire, and diagnosing the cause of fire, also useful in finding the way to approach the fire by the experiments we conducted. But it still had many things need to be improved in some aspects. For example, 1) not very useful to find the starting point of fire because there was not any information to show the location of the fire in the building; 2) Can't tell which floor the fire is located. Have to take long time to count 3) Can't see the details of each floor.

In order to improve 3D display, floor numbering, and more controls were added in a new prototype. How to show floor numbers and how to show the whole building, the building shape and details by 3D display were the main issues in this study. For

D. Harris (Ed.): Engin. Psychol. and Cog. Ergonomics, HCII 2007, LNAI 4562, pp. 157-163, 2007.

(c) Springer-Verlag Berlin Heidelberg 2007 
the 3D building display, the horizontal rotation ways and rotation speeds, and the ways of building tilting were tested and discussed.

\section{Method}

There are many different cognitive model in the interact between man and environment, different job-domain's characters and different control mode's requirements create the different interaction cognitive model [7]. To got the best mode of the 3D interface to fit the firefighter, we did these two experiment do detect many $3 \mathrm{D}$ display modes.

\subsection{Experimental Environment}

A new FID prototype (Version 2.0) has been developed as the experimental environment. All the fire scenarios were supposed to happen in 3 buildings.

Floor numbers were shown in 3D display in 5 different optional ways, and a new control for building rotation was added. Right now there are three ways to show building shape and detail by rotating, i.e. 1) rotate continuously at 2 optional speeds, 2) rotate step by step, and 3) rotate continuously and automatically, which means there was no way to control the rotation speed.

\subsection{Experimental Participants}

9 Fire firefighters aged from 24 to 28 years participated in our experiment. One of them has bachelor degree and one-year firefighting experience, and others have more than 5-year experience.

\subsection{Experiment 1}

\subsubsection{Experimental Design}

Five floor-numbering designs were tested in our test, the first way is putting floor numbers on the building wall; the second is putting numbers on two sides of the high rise; the third is putting them on building floors; the fourth is putting them on two sides of the high rise but the location of floor numbers keeps still while the building display keeps rotating; the fifth is similar as the second, but the floor numbers were shown in 3D mode. The purpose of this study was to compare the five designs and find which way is more efficiency and more favorable to firefighters.

\subsubsection{Experimental Tasks}

The hospital building and the tower building were used as stimulus background. Participants were asked to tell the floor number.

\subsubsection{Procedure}

The hospital building and the tower building were used as stimulus background. Each type of floor numbering was shown on one building. There were two test sequences of floor numbering shown in the table 1.For each experimental condition, several highlighted floor levels would be shown on the PC screen one by one. Participants were asked to tell the fired floor number as accurate and quickly as possible. 
Table 1. Two test sequences of floor numbering

\begin{tabular}{cc}
\hline Stimulus order 1 & Stimulus order 2 \\
\hline Wall-Hospital building & Fixed-Hospital building \\
Side—Tower building & Side-Tower building \\
Floor-Hospital building & Floor-Hospital building \\
Fixed-Tower building & Wall-Tower building \\
Cube-Hospital building & Cube-Hospital building \\
\hline
\end{tabular}

From the prototype interface, there were several ways to know which floor was currently highlighted. In order to avoid participants using other cues to find it, the left part of PC screen was masked by a piece of paper. And participants were asked to answer the floor number only by watching the rotating building.

\subsubsection{Experiment 1 Results}

Table 2 showed the percentage of correct responses and response time. It's found that showing number on floors was the worst way. It got the most errors, and took the longest time. (For the reaction time, $\mathrm{F}(4)=6.899, \mathrm{p}=0.00$; for the percentage of correct answer, $F(4)=7.939, p=0.00$.) Among the other four ways, there was no significant difference existed. But showing numbers on wall, and on two sides of building whatever fixed, or rotating with the building were better than the way of cube display.

Table 2. Percentage of correct responses and response time

\begin{tabular}{cccccc}
\hline & Wall & Side & Floor & Fixed & Cube \\
\hline $\begin{array}{c}\text { Percentage of } \\
\text { correct answers } \\
\text { Reaction time }\end{array}$ & $95 \%$ & $93 \%$ & $52.8 \%$ & $97.8 \%$ & $89.6 \%$ \\
$(\mathrm{~ms})$ & 1412.5 & 1555.5 & 11311.1 & 1344.4 & 2077.8 \\
\hline
\end{tabular}

The fig 1 showed firefighters' preference to the five kinds of floor numbering designs. It implied that firefighters' preference was consistent with the results of reaction time, and the percentage of correct answer. Showing numbers on floor got the least favorites. Cube way got the second least, and the other three were similar. 


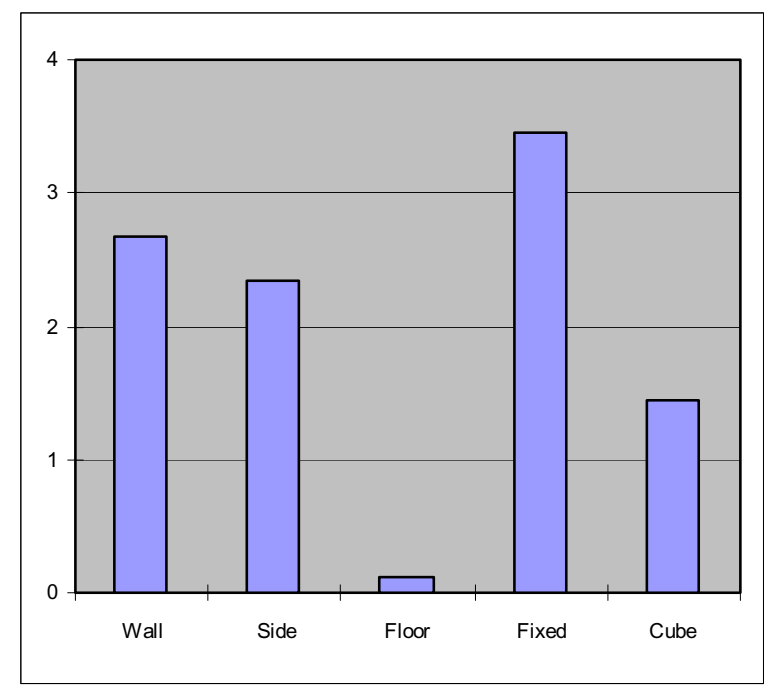

Fig. 1. Subjective ratings to different ways of floor numbering

From the three indexes we have got above, we would conclude that showing numbers on wall, or on two sides of building were better ways than showing them on floors. Going back to the behavior record, we found number shown on the floor was difficult to recognized. That's the reason people took longer time to tell the floor number. The reason why the first, second, and fourth ways got the similar performance could be that, because of the unregulated shape of hospital building, current interface was not able to show number on wall when the floor level was higher than 3. That made the three ways of showing number almost the same, so the performance and the subjective ratings under these three conditions were similar.

\subsection{Experiment 2}

\subsubsection{Experimental Design}

Right now the most popular way to show whole building in 3D mode is rotation. With the building rotating, people can see the building from different point of view. Nothing can be covered. There are two different rotation styles: one is rotating smoothly and continuously; the other is rotating step by step at a constant angle. People like building rotating continuously, but when the building structure is complex the programming costs of 3D rotation becomes much higher. So rotating step by step for high rise building is an alternative way to show all the structure details of it.

In this experiment, the rotation way was the independent variable including three levels: rotating continuously at two optional speeds, rotating step by step at two optional rotation angles, and rotating continuously at a fixed speed. First way allowed 
people choose one rotation speed from 1to10, which was called rotation "automatically"; Second way allowed people choose one rotation angle: 15o, or 30 o, and rotate the building by clicking the 150 , or 30 o button, which was called rotation "manually"; Third way was similar as the first one, but people were not allowed to control the rotation speed. It was fixed at 5. The third was called "none" in the setting menu of current prototype, which meant "automatically rotation at fixed speed".

\subsubsection{Experimental Tasks}

Each participant were asked to put out 18 fires including 6 single fires spread on single floor, 6 single fires spread on several floors, and 6 multi-fires (that meant there were several fire seeds in the building simultaneously). Half of the 18 fires were shown in 3D mode, the others shown in 3D with 2D floor plan.

For the way of rotation, 6 of the 18 fire scenarios were shown randomly in "auto" mode; 6 of them were shown in "manually" mode; the other 6 were shown in "automatic rotate at fixed speed" mode.

\subsubsection{Procedure}

For each fire scenario, participant was asked to accomplish three tasks: 1) find the starting point of the fire; 2) find the spread way; 3) find the path in the building for firefighter to put out the fire. Performance was recorded at three time points: 30 seconds, 90 seconds, and 5 minutes from the beginning when the fire was explored to participant. At each time point, the screen was suddenly black, and all the information was masked. Participant was asked to answer three questions: where was the fire? How did it spread? And how did you get to the fire to put it out? We counted the number of how many tasks were finished. Each of the three tasks was treated as one score. For example, if the participant only found the starting point of the fire at 30s time point, then found the spread of fire and the path to the fire at $90 \mathrm{~s}$ time point, the participant would get 1 score at 30s, 3 scores at 90s, and also 3 scores at 5 mins.

The reason why we recorded the performance at three time points was that, it's quite urgent when firefighters made a decision during a fire. Decision was usually made within one minute. If the interface prototype was good enough, it must be helpful at the first one, or two minutes. Therefore we set three time points: the first two time-points were for the measurement of efficiency, the third one was trying to investigate that, if firefighters had enough time, what kind of information they looked for to adjust, or confirm their decision.

\subsubsection{Experiment 2 Results}

2.4.4.1 The Way of Rotation. The way of rotation Table 3 showed how many scenarios, under three kinds of rotations, were finished at each time points. In the volume of task performance, ' 0 ' meant none of the three tasks was finished; ' 3 ' meant all of the three tasks were finished. 
Table 3. Number of people who finished the task successfully under different rotating conditions

\begin{tabular}{|c|c|c|c|c|c|}
\hline \multirow[t]{2}{*}{ Time point } & \multirow{2}{*}{$\begin{array}{c}\text { Task } \\
\text { performance }\end{array}$} & \multicolumn{3}{|c|}{ Rotate } & \multirow[t]{2}{*}{ Total } \\
\hline & & Auto & Manually & Auto (with fixed speed) & \\
\hline \multirow{4}{*}{$30 \mathrm{~s}$} & 0 & 5 & 6 & 3 & 14 \\
\hline & 1 & 22 & 12 & 12 & 46 \\
\hline & 2 & 10 & 8 & 4 & 22 \\
\hline & 3 & 17 & 23 & 30 & 70 \\
\hline \multirow[t]{2}{*}{ Total } & & 54 & 49 & 49 & 152 \\
\hline & 0 & 2 & 1 & & 3 \\
\hline \multirow[t]{3}{*}{$90 \mathrm{~s}$} & 1 & 5 & 4 & 4 & 13 \\
\hline & 2 & 9 & 3 & 1 & 13 \\
\hline & 3 & 38 & 41 & 44 & 123 \\
\hline \multirow[t]{2}{*}{ Total } & & 54 & 49 & 49 & 152 \\
\hline & 0 & 1 & & & 1 \\
\hline \multirow{3}{*}{$5 \mathrm{~min}$} & 1 & 3 & 1 & 1 & 5 \\
\hline & 2 & & 1 & & 1 \\
\hline & 3 & 50 & 47 & 48 & 145 \\
\hline Total & & 54 & 49 & 49 & 152 \\
\hline
\end{tabular}

Comparing with the people numbers at three time points, it's found that, at $30 \mathrm{~s}$, participants didn't finish all the tasks; but at 90s, and 5mins, almost all the people finished. It proved that firefighters only took one, or two minutes to make decision.

Comparing the three rotation conditions at three time points, people finished all three tasks under situation of automatic rotation at fixed speed were more than people under the other two conditions at 30s, and 90s. From the chi-square test, it's found the condition of "automatic rotation at fixed speed" at 30s was the most helpful for the decision efficiency, "automatic rotation with optional choice" the least helpful (30s:X2(6) $=11.057, \mathrm{p}=.087)$. There was no difference among the three conditions at 90s, and 5mins (90s:X2(6) =10.026, $\mathrm{p}=.124$ 5min:X2(6) $=5.304, \mathrm{p}=.505)$.

In this experiment, both the first and third conditions were automatic rotation. But the third one was better than "rotating manually", and the first worse. It looks strange. Only difference between the first and third condition was the 3D control. First condition allowed firefighters control the rotation speed, but third one didn't. It probably meant that the manual control part slowed down information acquirement and the process of decision making. 
2.4.4.2 Subjective Ratings to the Three Kinds of Rotations. From Table 4 we found 4 out of nine firefighters preferred automatic rotation with speed choices, another 4 of nine preferred rotating manually, only one of them like automatic rotation at a fixed speed. For the rotation speed and rotation angle, the choices were diverse. So, people's preference is not consistent with their performance.

Table 4. Preference to three kinds of rotating

\begin{tabular}{cccccccc}
\hline Horizontal rotation & $\begin{array}{c}\text { Rotate automatically } \\
\text { (speed is controlled } \\
\text { manually) }\end{array}$ & Rotate manually & $\begin{array}{c}\text { Rotate automatically } \\
\text { (speed is fixed) }\end{array}$ \\
\hline Number of people & & 4 & & & 4 & & 1 \\
\hline Speed of rotation & $1-5$ & $5-$ & 10 & 150 & 30 o & Both & \\
\hline Number of people & 1 & 2 & 1 & 2 & 1 & 1 & 1 \\
\hline
\end{tabular}

\section{Conclusion and Discussion}

In two experiments, we compare the five designs on floor number and compare building rotation way about the 3D building. We can make the following conclusions:

(1) Showing number on floors is the worst way. It got the most errors and took the longest time. Among the other four ways, there was no significant difference existed. But showing numbers on wall, and on two sides of building whatever fixed, or rotating with the building were better than the way of cube display.

(2) It's found the condition of "automatic rotation at fixed speed" at 30 s was the most helpful for the decision efficiency, "automatic rotation with optional choice" the least helpful. There was no difference among the three conditions at 90s, and 5mins.

\section{References}

1. Fang, Z.: Development of human-machine interaction: multimedia and multisensory. Human Factors 2, 34-38 (1998)

2. Li, X.: Research on Adaptive Human Computer Interface Doctoral Dissertation, Southwest Normal University, 1 (2004)

3. Everhart, A.: A Lesson in Campus Fire-Alarm System Design. Consulting-Specifying Engineer 1, 53-54 (2006)

4. Chen, Y., Bai, S., Liang, B.: HLA-based 3D Virtual Environment For Synthetic Simulation System. Computer Engineering and Applications 38, 21-24 (2002)

5. Wang, S.: Frame of Pattern Recognition Model Based on Cognitive Psychology. Editoral Board of Geomatics and Information Science of Wuhan University 27, 543-547 (2002)

6. Song, T., Liu, W.: Research of Cognitive-Based 3D Model for Visualization of Association Rule. Microcomputer Applications 1, 1-3 (2006)

7. Rasmussen, J.: Skills, rules, and knowledge; signals, signs, and symbols, and other distinctions in human performance models. IEEE Transactions on Systems, Man and Cybernetics, SMC-13, 257-266 (1983) 